\title{
Low insertion loss Modulator based on a vertically coupled Photonic Crystal Resonator
}

\author{
Kapil Debnath ${ }^{* a}$, Liam O’Faolain ${ }^{\mathrm{a}}$, Frederic Y Gardes ${ }^{\mathrm{b}}$, David Thomson ${ }^{\mathrm{b}}$, Graham Reed ${ }^{\mathrm{b}}$, Thomas \\ F. Krauss ${ }^{\text {a }}$ \\ ${ }^{a}$ SUPA, School of Physics and Astronomy, University of St Andrews, North St Andrews, Fife, \\ KY16 9SS UK \\ b Advanced Technology Institute, University of Surrey, Guildford, Surrey, GU2 7XH, UK
}

\begin{abstract}
We experimentally demonstrate a simple but more efficient technique to modulate and multiplex multiple WDM channels. Our design is based on a bus waveguide vertically coupled to multiple Photonic Crystal (PhC) resonator, each of which modulates an individual channel in place. The Photonic crystal resonator modulator provide very low switching energies ( $\sim \mathrm{JJ}$ ) while the bus waveguide can be made from a material with a low refractive index thereby allowing very efficient coupling with an optical fiber.
\end{abstract}

Keywords: silicon photonics, nanophotonics, photonic crystal cavity, optical modulator

\section{INTRODUCTION}

Wavelength division multiplexing (WDM) is a key technique for optical communications, maximizing the bit rate that may be transmitted over a single link. Due to its low cost and CMOS compatibility, silicon is a particularly attractive material for the implementation of WDM, enabling mass market applications such as fiber to the home and active optical cables $^{1}$.

Monolithic silicon based WDM systems typically consist of banks of components, e.g. modulators ${ }^{2}$ or laser/modulator combinationss $^{3}$, which are linked together by a wavelength multiplexer for coupling to a fiber. Such schemes suffer high losses at the interfaces between sections and the complexity, of the multiplexor in particular, requires very precise fabrication- both of which problems arise from the high refractive index of silicon. Coupled with the high power consumption of the Mach Zehnder Interferometer modulator, this makes the realization of Tbit/s systems very challenging.

Here, we demonstrate the principle of a much simpler yet more powerful system. Our design is based on a low index contrast bus waveguide (e.g. Silicon Oxynitride) vertically coupled to multiple Photonic Crystal (PhC) resonators, each of which modulates an individual channel. The $\mathrm{PhC}$ resonator has a very small mode volume, on the order of a cubic wavelength, and thus requires very little power to operate and occupies very little space. Additionally, due to the wavelength selective nature of the resonator, the channel may be modulated while in the bus, avoiding the need for complex multiplexors/demultiplexors. This allows everything to modulate an individual channel to be contained in an area of approximately 20um x 20um. As large arrays of single mode PhC cavities can be made, this system is very scalable and has potential for $1 \mathrm{Tbit} / \mathrm{s}$ data transmission.

*kd343@st-andrews.ac.uk 
Crucially, the vertical coupling technique reduces the problem of light insertion to that of coupling from a fibre into a large area, low refractive index waveguide. This circumvents the high insertion losses that are typical of high refractive index contrast nanophotonics, which otherwise require spot size converters or grating couplers. In this work, we achieve insertion losses of less than $3 \mathrm{~dB}$ using tapered fibers. By using techniques developed for Planar Lightwave Circuits, sub $1 \mathrm{~dB}$ insertion losses are possible ${ }^{4,5}$.

\section{FABRICATION}

The devices were fabricated in SOItec Silicon on Insulator material consisting of a 220nm top silicon layer on a 2000nm buried oxide layer. Using electron beam lithography the PhC designs are defined in the ZEP-520A layer and the pattern transferred into the silicon layer by reactive ion etching with a SF6/CHF3 gas mixture. To create the pin junction, doping windows were defined with "fingers" extending into the photonic crystal, similar to Ref. 6, see figure 1, and doped using the ion implantation of boron and phosphorous $\left(\sim 10^{19} / \mathrm{cm}^{3}\right)$. The gap between the $\mathrm{p}$ and $\mathrm{n}$ region is approximately $2 \mathrm{um}$. This separating region is slightly p-type doped with $\sim 10^{15} \mathrm{~B} / \mathrm{cm}^{3}$ (background doping of the as- bought SOI wafer). Alignment between the steps is carefully carried out by means of electron beam lithography. Doped silicon is known to be a source of optical losses; however, doping has been shown to be relatively insignificant in this system with Q-factors up to 40,000 observed for carrier densities of $10^{18} / \mathrm{cm}^{37}$.

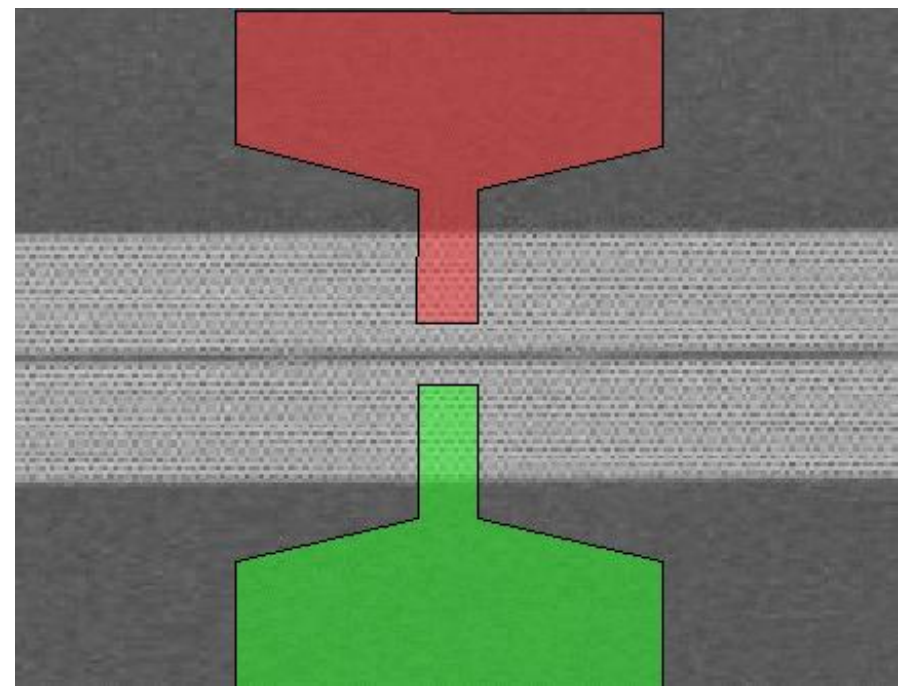

Figure 1 Image of the PhC resonator embedded in a pin junction. The p-doped region is shown schematically in red and the $n$-doped region in green. The PhC resonator is based on a width modulated line defect.

A SEM image of the device is shown in Fig. 1. The entire device is then covered with spin-on-glass FOx-14 (commercially available from Dow-corning) cladding. Although this process reduces the index contrast, it improves the CMOS compatibility of the final device. The design of high quality photonic crystals becomes somewhat more challenging ${ }^{8}$ but high $\mathrm{Q}$ resonators have be realized ${ }^{9}$. The oxide layer thickness is then reduced to $200 \mathrm{~nm}$ by RIE with $\mathrm{CHF}_{3}$ gas, which is found to be the optimum thickness for efficient coupling while maintaining high Q. In this work we used two different waveguide materials, Vias were etched through the oxide and Aluminum contacts are added using photolithography and thermal evaporation. Finally, $3 \times 2$ um SU8 bus waveguides were defined that run directly above the cavities, see figure 2 . 


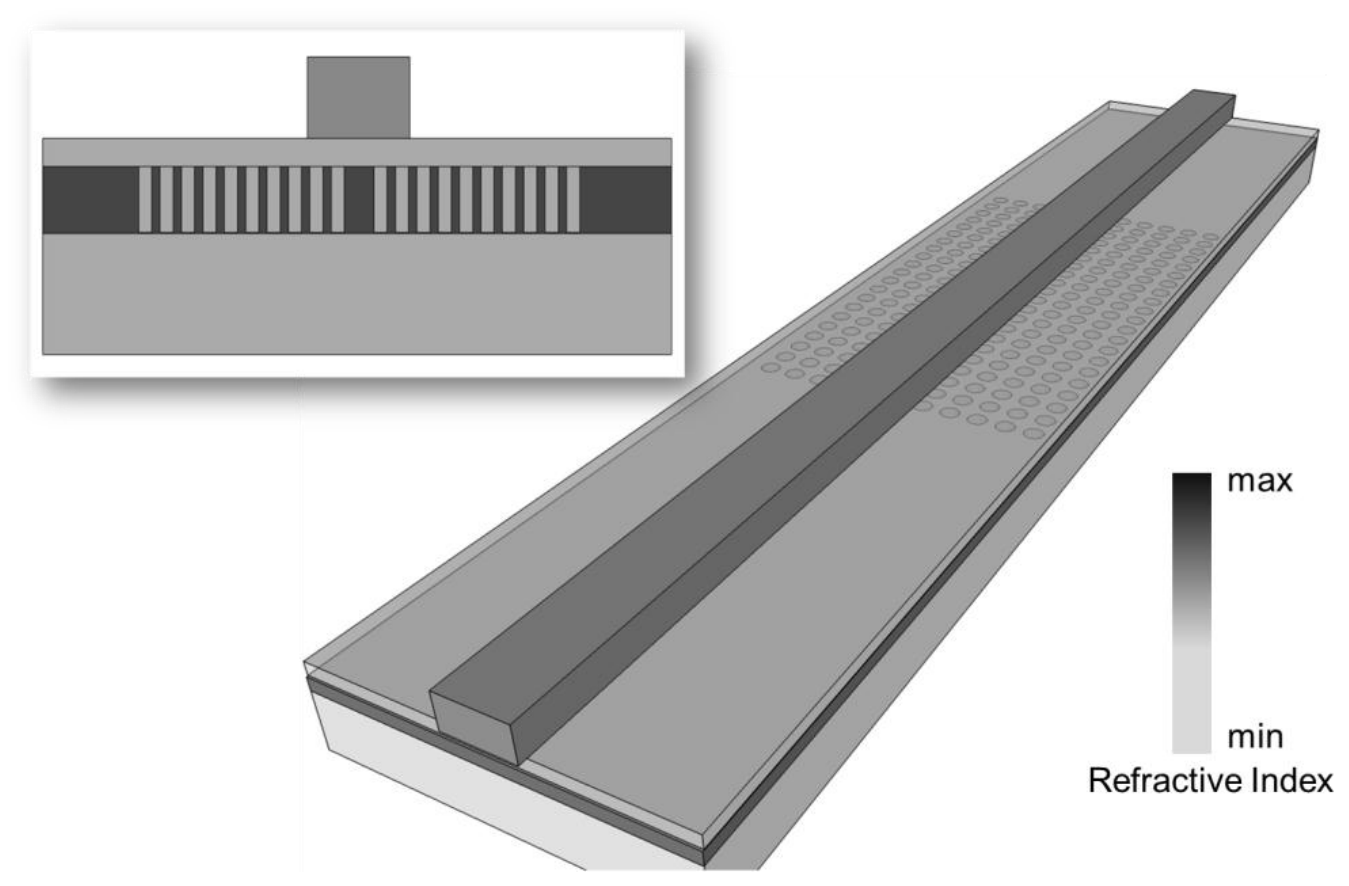

Figure 2: Schematic of the PhC resonator and bus waveguide (pin junction not shown).

\section{COUPLING}

To couple efficiently between two modes, two conditions must be satisfied- a spatial overlap must exist and their phases must match. The first is relatively easy to attain, but the second is very restrictive, generally requiring modes of similar effective refractive indices, as is used in most directional couplers. A solution lies in the small mode volume of a photonic crystal resonator. As this decreases, the corresponding k-vector distribution expands- an initial obstacle to the creation of high Q-factor resonators ${ }^{10}$ - and the problem of coupling reduces to that of matching the $\mathrm{k}$-vector space of the waveguide to that of a portion of the resonators. With a suitably designed $\mathrm{PhC}$ cavity, a low effective index mode may thus be matched to that of a silicon based resonator. 


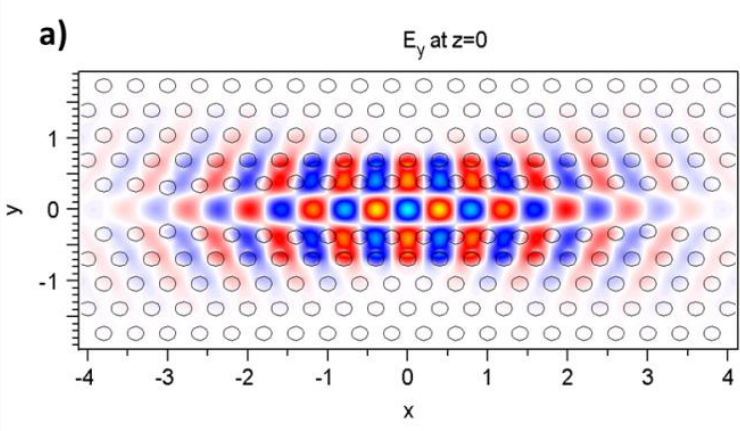

c)

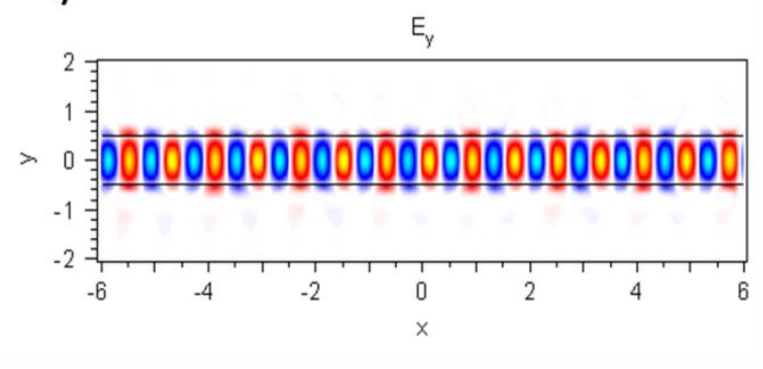

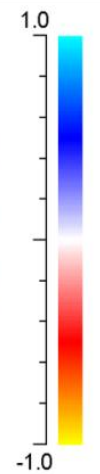
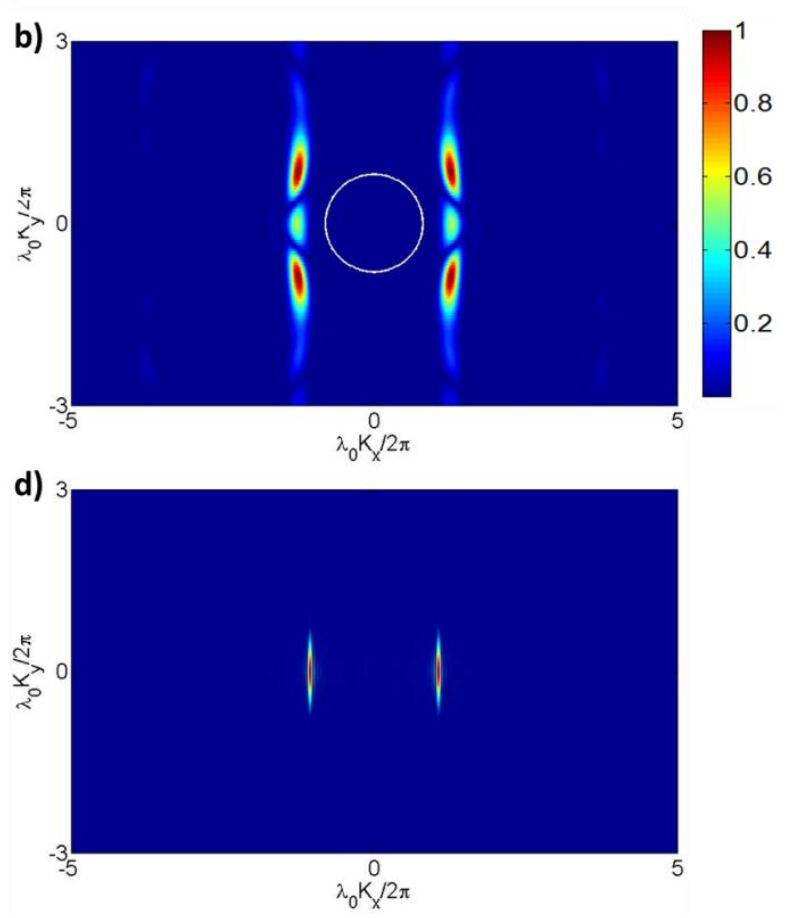

Figure 3: Illustration of the principle of $k$-space matching. The $E_{y}$ field plot of the photonic crystal cavity is shown in a) and the corresponding $k$-space in $b$ ). The $E_{y}$ field plot of the SU8 and corresponding $k$-space is shown in $c$ ) and d) respectively. To achieve coupling, there must be k-space overlap, i.e. the distribution in c) and d) should overlap.

This is a particularly powerful means of coupling to a photonic crystal resonator. Following the analysis of ${ }^{11}, 30 \mathrm{~dB}$ extinction ratios may be expected for a resonator with a $\mathrm{Q}$-factor or 10,000 - to date.

Not only may very high efficiencies be attained, but the bus waveguide creates the minimum perturbation to the resonator mode. In contrast, the popular in-plane bus $\mathrm{PhC}$ waveguides introduce boundaries to the resonator that introduce additional $\mathrm{k}$-vector components that lie inside the light cone $^{12}$, comprising the resonator Q-factor. Furthermore, efficient channel dropping is generally a difficult operation in $\mathrm{PhCs}$, requiring an undesirable design complexity, such as the heterostructure mirror ${ }^{13}$ which limits the useable wavelength range. Vertically coupling to single mode cavities potentially allows the use of 100s of channels over the full $\mathrm{C}$ and $\mathrm{L}$ bands. Fluctuations in resonance wavelength due to disorder have been shown to be within acceptable limits ${ }^{14}$.

Photonic crystal resonators are particularly attractive for the creation of high speed optical modulators ${ }^{6}$. The ultra-small mode volume makes them one of the most power efficient types of modulator. By using Q-factors greater than 10,000, it will be possible to achieve modulation with the small refractive index changes that available with carrier depletion, the fastest and most efficient mechanism available.

Figure 5 shows the modulation of a vertically $\mathrm{PhC}$ modulator. In this case, carrier injection is used to produce the high speed change in refractive index. As may be seen in fig. $5 \mathrm{~b}$, this is sufficient to shift the PhC resonator on and off resonance with the light source. As this is a $\mathrm{CW}$ measurement, carrier injection results in heating that redshifts the cavity resonance. Figure $5 \mathrm{~b}$ shows the high speed modulation of this device. The maximum modulation speed was $1 \mathrm{GBit} / \mathrm{s}$, which was limited by the design of the electrical contacts. Further optimization will increase the electrical efficiency of the device. The ultimate limit on the modulation speed will be given by the photon lifetime in the cavity. For a Q-factor of 10,000 , this limit is well in excess of $25 \mathrm{Gbit} / \mathrm{s}$. 

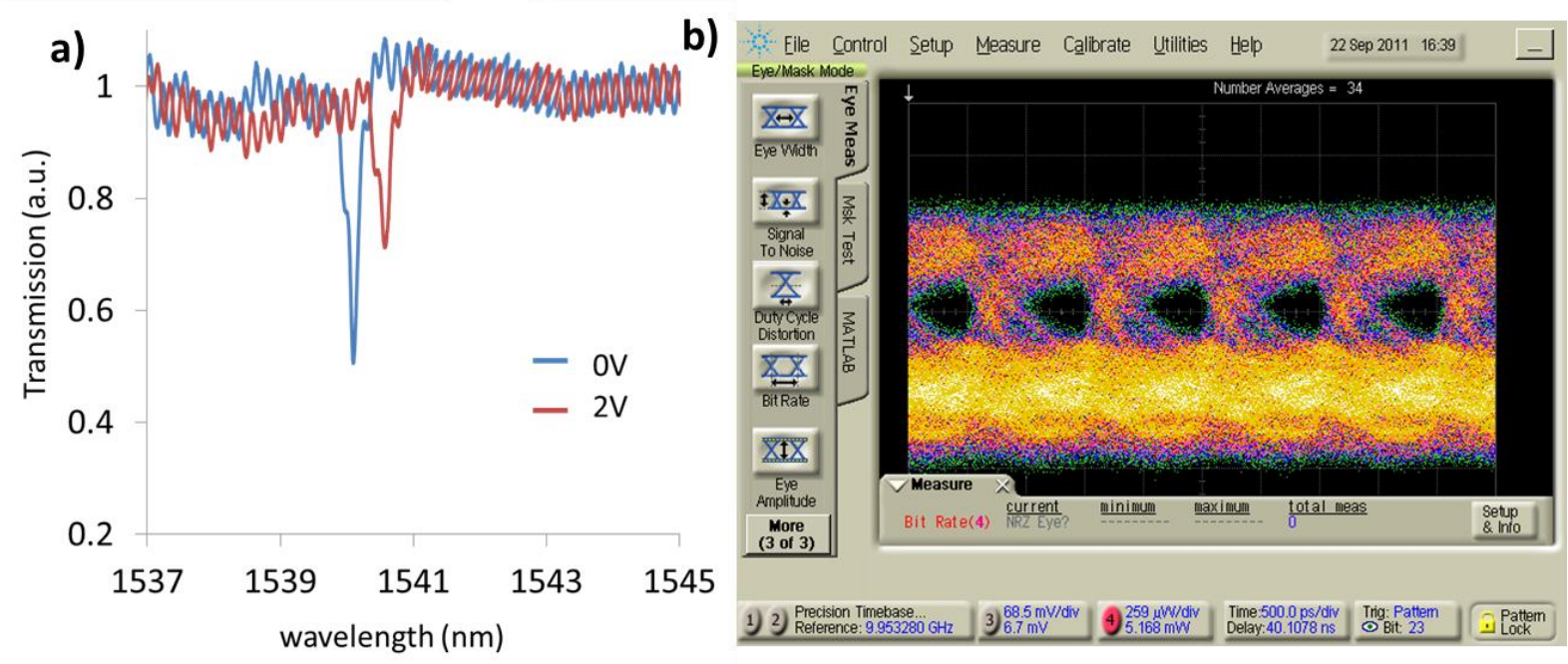

Figure 5 a) CW measurement of a PhC resonator modulator. The resonator $Q$-factor is 10,000. The estimated $\Delta n$ is $4 \times 10^{-4}$. The extinction ratio is relatively low due to non-optimum coupling. $b$ ) Eye diagram corresponding to modulation at $1 \mathrm{Gbit} / \mathrm{s}$. On this device, the modulation is limited by the contact design.

As a result of the relatively large dimensions of the bus waveguide $(3 \times 2 u m)$ and the low refractive index $(\mathrm{n}=1.56)$ of SU8, very efficient coupling is possible using a lensed fibre. In this initial demonstration, the fiber-to-fiber insertion loss was $2.8 \mathrm{~dB}$. Using more advanced techniques such as cantilever couplers ${ }^{15}$, significant reductions are possible.

\section{CONCLUSION}

We demonstrate the operating principle of a new configuration for optical modulators. Through the use of photonic crystal modulators vertically coupled to a low index contrast bus waveguide- high speed, low power consumption, low insertion loss modulation may be achieved on a very small footprint. The technique of vertical coupling is the key breakthrough that eliminates the need for complex, fault intolerant components such as Arrayed Waveguide Gratings while simultaneously providing highly efficient coupling to an optical fiber. This compares very favorably with modulation systems reported in the literature and marks an important breakthrough in this field.

\section{REFERENCES}

[1] Analui, B., Guckenberger, D.; Kucharski, D. and Narasimha, A., "A Fully Integrated 20-Gb/s Optoelectronic Transceiver Implemented in a Standard 0.13-um CMOS SOI Technology," IEEE Journal of Solid State Circuits 41, 2945-2955 (2006)

[2] Chen, L., Doerr, C. R., Dong, P., Chen, Y-k, "Monolithic silicon chip with 10 modulator channels at 25 Gbps and 100-GHz spacing," Optics Express 19, B946-B951 (2011)

[3] Jain, S. R., Sysak M. N., Kurczveil G. and Bowers J. E., "Integrated hybrid silicon DFB laser-EAM array using quantum well intermixing," Optics Express 19, 13692-13699 (2011)

[4] Larsen, B., Nielsen, L., Zenth, K., Leick L., Laurent-Lund, C., Andersen, L. and Mattsson K., "A Low-Loss, Silicon-Oxynitride Process for Compact Optical Devices," Proceedings of ECOC (Rimini, Italy, 2003).

[5] Hibino, Y., "Recent Advances in High-Density and Large-Scale AWG Multi/Demultiplexers With Higher IndexContrast Silica-Based PLCs," Lightwave Technology, Journal of 8, 1090-1101 (2002)

[6] Tanabe, T., Nishiguchi, K., Kuramochi, E. and Notomi, M., "Low power and fast electro-optic silicon modulator with lateral p-i-n embedded photonic crystal nanocavity," Optics Express 17, 22505-22513 (2009)

[7] Cardile, P, Franzò G., Lo Savio, R., Galli M., Krauss, T. F., Priolo F, and O' Faolain, L, "Electrical conduction and optical properties of doped silicon-on-insulator photonic crystals," Applied Physics Letters 98, 203506 (2011) 
[8] White, T. P., O'Faolain, L., Li, J., Andreani, L. C. and Krauss, T. F., "Silica-embedded silicon photonic crystal waveguides," Optics Express 16, 17076-17081 (2008)

[9] Jeon, S-W., Han, J-k, Song, B-S and Noda, S., "Glass-embedded two-dimensional silicon photonic crystal devices with a broad bandwidth waveguide and a high quality nanocavity," Optics Express 18, 19361-19366 (2010).

[10] Akahane, Y., Asano, T., Song, B. S. and Noda, S., "High-Q photonic nanocavity in a two-dimensional photonic crystal," Nature 425, 944-947 (2003)

[11] Xu, Y., Li, Y., Lee, R K., and Yariv, A., "Scattering-theory analysis of waveguide-resonator coupling," Physical Review E 62, 7389-7404 (2000)

[12]Zhang, Z. and Qiu M., "Compact in-plane channel drop filter design using a single cavity with two degenerate modes in 2D photonic crystal slabs," Optics Express 13, 2596-2604 (2005)

[13] Takano, H., Song, B. S., Asano, T., Noda, S., "Highly efficient multi-channel drop filter in a two-dimensional hetero photonic crystal," Optics Express 14, 3491-3496 (2006)

[14] Portalupi, S. L., Galli, M., Belotti, M., Andreani, L.C., Krauss T. F. and O'Faolain, L., "Deliberate versus intrinsic disorder in photonic crystal nanocavities investigated by resonant light scattering," Physical Review B 84, 045423 (2011) 